\title{
Elastic strain energy density decomposition in failure of ductile materials under combined torsion-tension
}

\author{
Nikos P Andrianopoulos ${ }^{*+}$ and Vasileios M Manolopoulos ${ }^{\dagger}$
}

\begin{abstract}
The constitutive behavior and failure of ductile materials are described in the present work for a general case of loading in terms of the secant moduli, which depend on the first (dilatational) and second (deviatoric) strain invariants. This approach exposes the distinct behavior of materials to the equivalent normal and shear stresses. The secant moduli enable the establishment of two (instead of one) constitutive equations necessary for the complete description of these materials. Emphasis is given in the accuracy of the resulting constitutive equations in terms of their predictions relative to actual experimental data for two materials systems. Failure predictions, according to T-criterion, are derived for two materials under combined torsion and tension, which are in good agreement with experimental data. Finally, the associated failure surfaces in a stress space are presented as well.
\end{abstract}

Keywords: Elastic strain energy density; Nonlinear elastic deformations; Combined stress paths; Constitutive equations; T-criterion

\section{Background}

The importance of strain energy density and in particular the distortional part of it relative to material failure was understood, conjectured and postulated, as early as in the era of Maxwell (1937) and others (Hencky 1924; Huber 1904). Strain energy, supplied to the material via some generalized loading, can be additively decomposed into two parts: the elastic and the inelastic one (for metals the plastic one); the elastic strain energy is recoverable upon removing the load (i.e., the corresponding strain at the unloaded state is zero) due to the fact that the materials remained elastic throughout its loading; the plastic strain energy is unrecoverable (and sometimes is called plastic work because it represents the energy lost to microstructural dissipative processes such as grain boundary slippage) that, when the load is removed, yields a permanent strain known as plastic strain.

Many failure criteria have been proposed and used to predict the initiation of macroscopic material failure under various loading conditions (Li 2001). In Christensen's

\footnotetext{
* Correspondence: nandrian@central.ntua.gr

${ }^{\dagger}$ Equal contributors

Department of Mechanics, Faculty of Applied Sciences, National Technical University of Athens, 5 Heroes of Polytechnion Avenue, GR 15773 Athens, Greece
}

failure theory (Christensen 2014), failure represents the termination of elastic behavior and not plastic behavior. In our failure theory, failure is defined as the loss of the ability of the material to store elastic strain energy. This may happen at the familiar yield point (elastic - perfectly plastic materials), or at maximum engineering loading point (hardening materials) or just at the moment of fracture (brittle materials). In this sense, the present definition of failure covers all the as above cases.

Phenomena are materialized in expenses of available resources. In case of failure of materials, the only available resource is elastic strain energy, stored into the material through elastic (linear or not) deformations. Elastic deformations exist from the very first load step until the last one. From this point of view, the amount of plastic work has no causative role in failure because it is the outcome of the action of available strain energy. Plastic work is a result of this action and cannot be the cause of other processes, including failure. It is a type of specific failure per se. This specific failure may be complete (plastic collapse), driving to the final loss of the ability of the material to store elastic strain energy when the material is elastic - perfectly plastic, implying saturation of the Bauschinger loop. Otherwise, in the so-called hardening 
materials (where both elastic and plastic strains coexist), the material may fail by either brittle or ductile fracture as it will be discussed in the sequel.

It is well known (Bao and Wierzbicki 2004) that plastic strains can depend on pressure. There are loading conditions (such as torsion) where in the absence of pressure plastic strains are dominant, or they are negligible when hydrostatic pressure prevails. Failure criteria based exclusively on stresses cannot capture the differentiation between elastic and plastic strains since there is no 'elastic' or 'plastic' differentiation for the stresses.

Concerning nonlinear elastic deformations, it is true that they, usually, can be neglected in engineering applications for practical reasons. However, all materials (especially brittle) show a degree of nonlinearity deserving consideration. Nevertheless, the strongest reason to incorporate nonlinear elastic deformations is the hardening behavior of materials, where both elastic and plastic strains coexist. In the hardening area, the nonlinearity of elastic deformations is certain.

A classical criterion marking pressure dependence is the Coulomb (Heyman 1997) criterion, which after Mohr (1914) states that a material fails when 'a proper combination of shear and normal stresses is realized.' This heuristic statement is logically perfect but lacks a quantitative description as far as it is based on experimental data interpolation and/or arbitrary assumptions for the shape of the failure envelope. In addition, it exhibits problematic behavior for tensile stresses (e.g., tension cut-off). Many failure criteria of this type exist in the open literature (Paul 1968; Stassi 1967; Schajer 1998; Bigoni and Piccolroaz 2004; Mahendra and Bhawani 2012; Drucker and Prager 1952), the simplest being the original Mohr-Coulomb criterion $\tau+a \cdot \sigma=c$.

All these criteria have two points in common:

(a) They are based on formulations involving a single function comprising the sum of two parts, one involving an expression of the shear stresses and the one involving the contribution of the normal stresses.

(b) They are based on stresses only and, especially, on those lying on the 'critical' plane of extreme stresses $\left(\sigma_{1}, \sigma_{3}\right)$. It is difficult to accept that the intermediate stress $\sigma_{2}$ plays no role, even in the critical plane.

An interesting exception is the criterion that was developed by Christensen (1997, 2004), where the dilatational and deviatoric (distortional) parts of the elastic stress tensor are introduced. This approach allows for a direct association of the decomposed parts of the stress tensor with the respective geometric changes activated during loading, i.e., volume (lengths) changes and shape (angles) changes, which are the only possible geometric changes in a deformable solid.

A criterion based on elastic strain energy density in case of linear elasticity was the first version of the socalled T-criterion which has been proved adequate to predict failure conditions for pre-cracked (Theocaris and Andrianopoulos 1982a, b) or uncracked geometries (Andrianopoulos 1993; Andrianopoulos and Boulougouris 1994). It was based on the von Mises's (1913) criterion and an addendum giving an answer to the question: "What happens with the, not covered by von Mises, dilatation of the material?' However, this early version of the T-criterion was unable to give an answer to the phenomenon of 'pressure dependence' of failure.

In order to cover pressure dependence and nonlinearity of elastic strains, a generalization of the T-criterion was recently introduced (Andrianopoulos and Boulougouris 2004; Andrianopoulos et al. 2007, 2008; Andrianopoulos and Manolopoulos 2012). The general case of an isotropic material showing nonlinear elastic behavior was considered, and the total elastic strain energy density $T$ was anticipated as the characteristic quantity for the respective conservative field. This quantity $T$ is path independent and so, a relationship between dilatational $T_{\mathrm{V}}$ and distortional part $T_{\mathrm{D}}$ of $T$ is obtained (Andrianopoulos and Manolopoulos 2010). This approach proved to be quite successful in predicting the failure behavior of metals under high levels of pressure (Andrianopoulos and Manolopoulos 2012) where for the first time - according to our best knowledge - the classical experiments of Bridgman (1952) were theoretically justified.

The goal of this paper is to give a different view of what a constitutive equation is and to emphasize the idea that the prediction of failure of a material is mainly equivalent to the problem of deriving the proper constitutive equations. Also, the thoughts, the assumptions and the considerations that made the writers to generalize T-criterion are presented in detail. The new generalized form of T-criterion, which is used in this work, was thoroughly explained and described in (Andrianopoulos and Manolopoulos 2012). Now the application of T-criterion is examined for combined loading paths of two ductile materials. Two sets of data with various combined tension-torsion loading paths are studied (Ali and Hashmi 1999; Marin 1948). The aim is to test T-criterion's forecast for these loading paths.

\section{Methods}

\section{Constitutive equations}

The constitutive behavior of elastic materials is described by an equation relating equivalent stress to equivalent strain. The simplest and, perhaps, the more reasonable 
way to define these quantities is the von Mises yield criterion (von Mises 1913) according to which

$$
\left.\begin{array}{l}
\sigma_{\mathrm{eq}}=\frac{1}{\sqrt{2}}\left[\left(\sigma_{1}-\sigma_{2}\right)^{2}+\left(\sigma_{2}-\sigma_{3}\right)^{2}+\left(\sigma_{3}-\sigma_{1}\right)^{2}\right]^{1 / 2} \\
\varepsilon_{\mathrm{eq}}=\frac{1}{1+v} \frac{1}{\sqrt{2}}\left[\left(\varepsilon_{1}-\varepsilon_{2}\right)^{2}+\left(\varepsilon_{2}-\varepsilon_{3}\right)^{2}+\left(\varepsilon_{3}-\varepsilon_{1}\right)^{2}\right]^{1 / 2}
\end{array}\right\}
$$

where $\sigma_{i}$ and $\varepsilon_{i}$ are the principal stresses and strains, respectively, and $v$ is the Poisson ratio.

Then, the required constitutive equation is a relation $\sigma_{\text {eq }}=f\left(\varepsilon_{\text {eq }}\right)$. This equation reflects the behavior of a material under shear stresses causing only shear strains, which, in turn - by the von Mises assumption - are responsible for its plastic behavior. The von Mises yield criterion is not designed to predict failure under equal hydrostatic pressure. This is easily understood by inspecting Equation (1) where for $\sigma_{1}=\sigma_{2}=\sigma_{3}$, it is obtained that $\sigma_{\text {eq }}=0$ and $\varepsilon_{\text {eq }}=0$. Then, strain energy density, i.e., the area under the curve $\sigma_{\text {eq }}=f\left(\varepsilon_{\text {eq }}\right)$, vanishes under equal hydrostatic pressure, in case when Equation (1) is solely used. Consequently, any constitutive equation involving the effective stresses and effective strains as defined by Equation (1) cannot describe the behavior of the material completely. Therefore, some expression in terms of the normal stresses and strains is required in order to capture the hydrostatic contribution to the material behavior. The natural choice for such expressions is the hydrostatic pressure $p$ and volumetric change $\Theta$, according to

$$
p=\left(\sigma_{1}+\sigma_{2}+\sigma_{3}\right) / 3, \Theta=\varepsilon_{1}+\varepsilon_{2}+\varepsilon_{3}
$$

If one assumes that a constitutive relation of the form $p=p(\Theta)$ exists, then the area under the curve representing this constitutive relation represents the dilatational strain energy density stored into the material.

The existence of two components of the strain energy density, one for the dilatation and one for the distortion of the material behavior, suggests that the constitutive behavior could also be considered as consisting of two parts, one responsible for the dilatation (in terms normal stresses/strains) and the other for the distortion (in terms of shear stresses/strains) of the material. In turn, this suggests that it is natural to introduce two constitutive equations instead of one for describing completely the behavior of a material, provided it can be proved that the two equations are equivalent to the one produced by the differentiation of the total strain energy density with respect to the strains. Figure 1 shows the potential graphs of these two equations, namely, $p=p(\Theta)$ and $\sigma_{\text {eq }}=\sigma_{\text {eq }}\left(\varepsilon_{\text {eq }}\right)$ for an arbitrary material, with linear, nonlinear elastic, and hardening behavior.

Essential to our analysis are the following remarks with respect to Figure 1:

1. By definition, strains in Figure 1a must be elastic as dictated by von Mises criterion (von Mises 1913). The stress-strain relationship is linear up to point $\mathrm{A}$ and generally nonlinear afterwards. Direct experimental data for $p=p(\Theta)$ curves are not available as triaxial tension is not easily accomplished. Usually, uniaxial tension data are used and an experimental or 'graphical' unloading is performed in order to find the elastic part of strain, i.e., the strain that can still be attributed to elastic response. Another option is from a graphical unloading of the curve $p=p(\Theta)$. These two alternatives are described thoroughly in Appendix 1.

2. Plastic strains appear in Figure $1 \mathrm{~b}$ after the end of both linear (point A) and nonlinear (point L) elasticity, although elastic strains continue
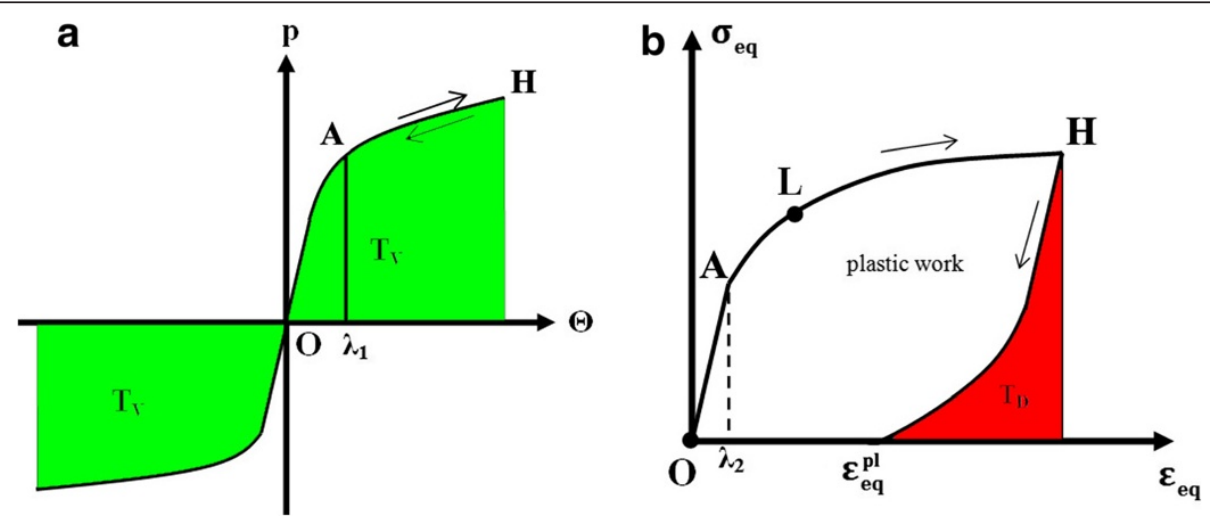

Figure 1 Constitutive equations of an arbitrary material in the engineering space. (a) $p=p(\Theta)$. Strains are assumed elastic. Unloading is not required. The two branches for $\pm \Theta$ are not necessarily symmetric. (b) $\sigma_{\text {eq }}=\sigma_{\text {eq }}\left(\varepsilon_{\text {eq }}\right)$. Unloading is required. Unloading line is arbitrary. 
developing up to the end (point $\mathrm{H}$ ), in case of hardening materials.

3. Both equations $p=p(\Theta)$ and $\sigma_{\mathrm{eq}}=\sigma_{\mathrm{eq}}\left(\varepsilon_{\mathrm{eq}}\right)$ are essential for the description of a material. They are considered as unique for each material and contain information about the energy balance and the type of failure. The traditional representation of materials through the second of them only (i.e., $\sigma_{\text {eq }}=\sigma_{\text {eq }}\left(\varepsilon_{\text {eq }}\right)$ ) and von Mises failure are incomplete.

4. In each curve of the above plots, a critical point can be located, marking the end of the development of elastic strains and, so, the collapse of the respective constitutive equation. The area of the graph under the loading part of the constitutive response line for any given level of strain on the horizontal access represents the value of the stored energy density. The elastic part of this energy density is represented by the area to the right of the unloading line when the stress returns to zero and the vertical from the strain reached for the terminal point on the curve. By definition, the terminal point in Figure 1a is the critical one when no plastic strains appear in this plot (see above remark 1). Consequently, the whole area from zero to terminal point in Figure 1a represents elastic strain energy density $T_{\mathrm{V}}$ stored for volume changes. Respectively, the unloading in Figure $1 \mathrm{~b}$ from the terminal point $\mathrm{H}$ is a line (straight or not is unconcerned) ending at point $\left(\varepsilon_{\mathrm{eq}}^{\mathrm{pl}}, 0\right)$ whose abscissa represents the unrecoverable plastic strain. This line separates the whole area into available elastic strain energy density $T_{\mathrm{D}}$ and plastic work.

5. The two branches of curve $p=p(\Theta)$ are not symmetric with respect to the origin of axes, especially in case of brittle materials. However, the area under each branch $\left(T_{\mathrm{V}}\right)$ is the same when the terminal point of each branch coincides with the critical one.

6. The geometry of the specimen and the current stress state at any given point of the specimen affect the velocity of a 'pointer' running along these two curves. When normal stresses/strains prevail, the velocity of the pointer on $p=p(\Theta)$ is higher than that on $\sigma_{\mathrm{eq}}=\sigma_{\mathrm{eq}}\left(\varepsilon_{\mathrm{eq}}\right)$, the opposite being true when shear stresses/strains prevail. Then, according to the specimen geometry and the stress state, the pointer on one curve arrives at the respective critical point before its companion does on the other curve, marking the type of failure.

A specific form of the abovementioned constitutive equations can be derived for the case of an arbitrary material by introducing the first strain and second deviatoric strain invariants $I_{1}, J_{2}$, respectively, and the secant elastic bulk modulus $K_{\mathrm{S}}$ and secant elastic shear modulus $G_{S}$ where

$$
\begin{aligned}
& I_{1}=\varepsilon_{1}+\varepsilon_{2}+\varepsilon_{3}=\Theta, \\
& \sqrt{J_{2}}=\frac{1}{\sqrt{6}}\left[\left(\varepsilon_{1}-\varepsilon_{2}\right)^{2}+\left(\varepsilon_{2}-\varepsilon_{3}\right)^{2}+\left(\varepsilon_{3}-\varepsilon_{1}\right)^{2}\right]^{1 / 2}
\end{aligned}
$$

Then, the complete behavior of a material as a function of $I_{1}$ and $J_{2}$ is described by

$$
K_{\mathrm{S}}=K_{\mathrm{S}}\left(I_{1}, J_{2}\right), \quad G_{\mathrm{S}}=G_{\mathrm{S}}\left(I_{1}, J_{2}\right)
$$

Equation (4) constitutes the necessary minimum set of constitutive equations for an isotropic material replacing Equations (1) and (2). Now, quantities $T_{\mathrm{V}}$ and $T_{\mathrm{D}}$ can be estimated as we are describing in the subsequent analysis. Referring to Figure 1a, the dashed green area in the first or third quadrant equals to dilatational strain energy density $T_{\mathrm{V}}$ and it is solely elastic. For the estimation of elastic distortional strain energy density $T_{\mathrm{D}}$, the respective red dashed area in Figure 1b provides the necessary value while the remaining area under the curve represents plastic work. Depending on the type of failure, i.e., brittle fracture (cleavage) or plastic flow (slip), either $T_{\mathrm{V}}=T_{\mathrm{V}, 0}$ or $T_{\mathrm{D}}=T_{\mathrm{D}, 0}$ is satisfied, where $T_{\mathrm{V}, 0}, T_{\mathrm{D}, 0}$ are the critical elastic strain energy densities.

\section{Failure criterion}

Given the partitioning of the elastic strain energy density to two parts (i.e., distortional and dilatational), the proposed failure criterion for the general case of nonlinearity is introduced as

$$
\begin{aligned}
& T_{\mathrm{V}}=\int_{0}^{I_{1,0}} \frac{1}{2} K_{\mathrm{S}}\left(I_{1}, J_{2}\right) d I_{1}^{2}=T_{\mathrm{V}, 0} \text { for failure by cleavage } \\
& T_{\mathrm{D}}=\int_{0}^{J_{2,0}} 2 G_{\mathrm{S}}\left(I_{1}, J_{2}\right) d J_{2}=T_{\mathrm{D}, 0} \text { for failure by slip }
\end{aligned}
$$

Equation (5) constitutes the general form of the T-criterion (Andrianopoulos and Boulougouris 1994), according to which

(A) Failure by cleavage (brittle fracture) occurs when $T_{\mathrm{V}}$ reaches a critical value $T_{\mathrm{V}, 0}$

(B) Failure by slip (plastic flow) occurs when $T_{\mathrm{D}}$ reaches a critical value $T_{\mathrm{D}, 0}$.

To quantify the failure behavior of an elastic material described by Equation (4), the evaluation of upper integration limits $I_{1,0}$ and $J_{2,0}$ for the quantities $I_{1}$ and $J_{2}$ appearing in Equation (5) is postulated. 
A relationship between secant elastic moduli and strain invariants is obtained which, as it is shown in Appendix 2 , in the present case takes the form:

$$
\frac{\partial\left(I_{1} K_{\mathrm{S}}\right)}{\partial J_{2}}=2 \frac{\partial G_{\mathrm{S}}}{\partial I_{1}}
$$

Thus, Equations (5) and (6) constitute a system of three equations with four unknowns, $K_{\mathrm{S}}\left(I_{1}, J_{2}\right), G_{\mathrm{S}}\left(I_{1}, J_{2}\right)$, $I_{1,0}$, and $J_{2,0}$. One of the functions $K_{\mathrm{S}}$ or $G_{\mathrm{S}}$ can be given experimentally, and subsequently the system can be solved for any prescribed loading path and the proposed criterion can be applied. Required constants, like $\left(\lambda_{1}, \lambda_{2}\right)$ or $\left(K_{\mathrm{S}}^{0}, G_{\mathrm{S}}^{0}\right)$ can be obtained from Figure 1.

\section{Results and discussion}

Two sets of experimental data are used to verify the present theoretical predictions. The first one (Ali and Hashmi 1999) gives detailed description of the material (constitutive equations, type of failure, etc.) and permits a rigorous examination of its results. The second one is a classical series of data presented by Marin (1948).

\section{Application to En8 steel}

In this section we will be using the experimental results originally presented in (Ali and Hashmi 1999) for En8 (BS 970) steel. All the necessary information concerning material properties, loading paths, and failure conditions is given. The lack of hydrostatic tension or compression data is a barrier in defining the exact form of $p=p(\Theta)$ and the exact value of the critical dilatational strain energy density $T_{\mathrm{V}, 0}$. The required information was obtained under uniaxial tension conditions according to the procedure described in Appendix 1.

The experimental procedure, described in (Ali and Hashmi 1999) involved the application of combined torsion-tension loading under controlled conditions. Two types of loading paths were investigated:

The first loading path was torsion within the elastic range of the material and then axial tension beyond the uniaxial yield stress $\sigma_{\mathrm{Y}}(=600 \mathrm{MPa})$ holding the initial angle of twist constant.

The second loading path was tension within the elastic range of the material and then torsion beyond the torsion yield stress $\tau_{\mathrm{Y}}(=350 \mathrm{MPa})$ holding the initial axial displacement constant.
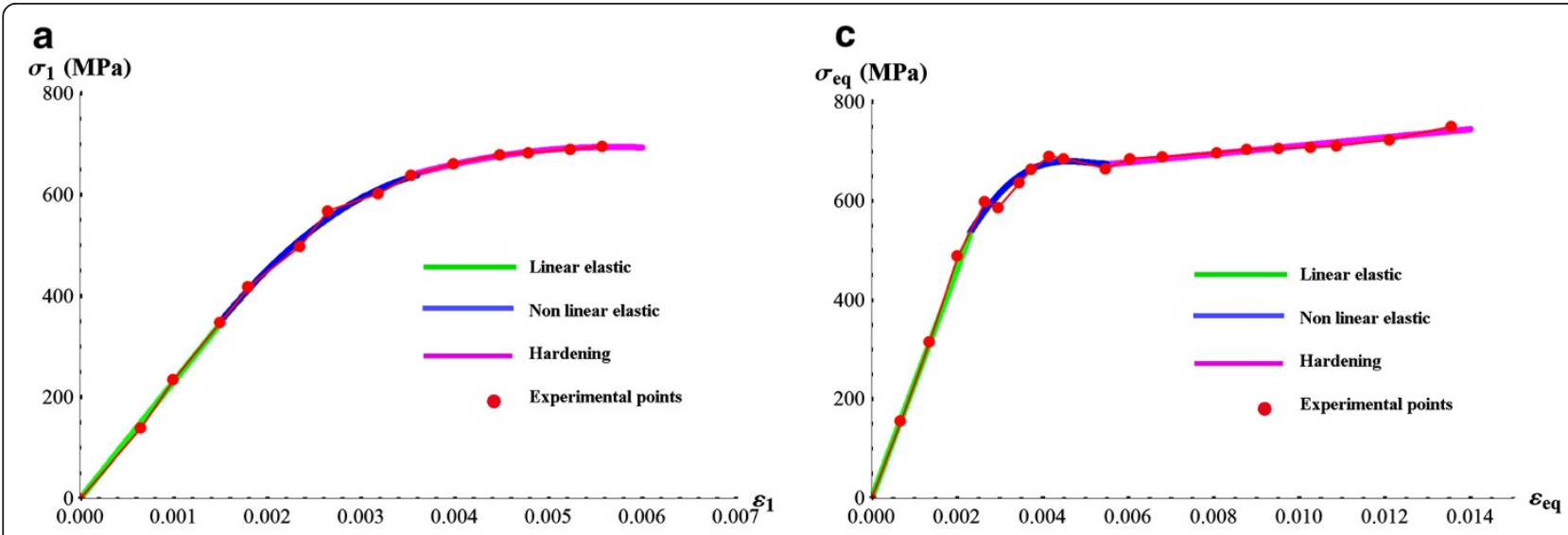

b

d
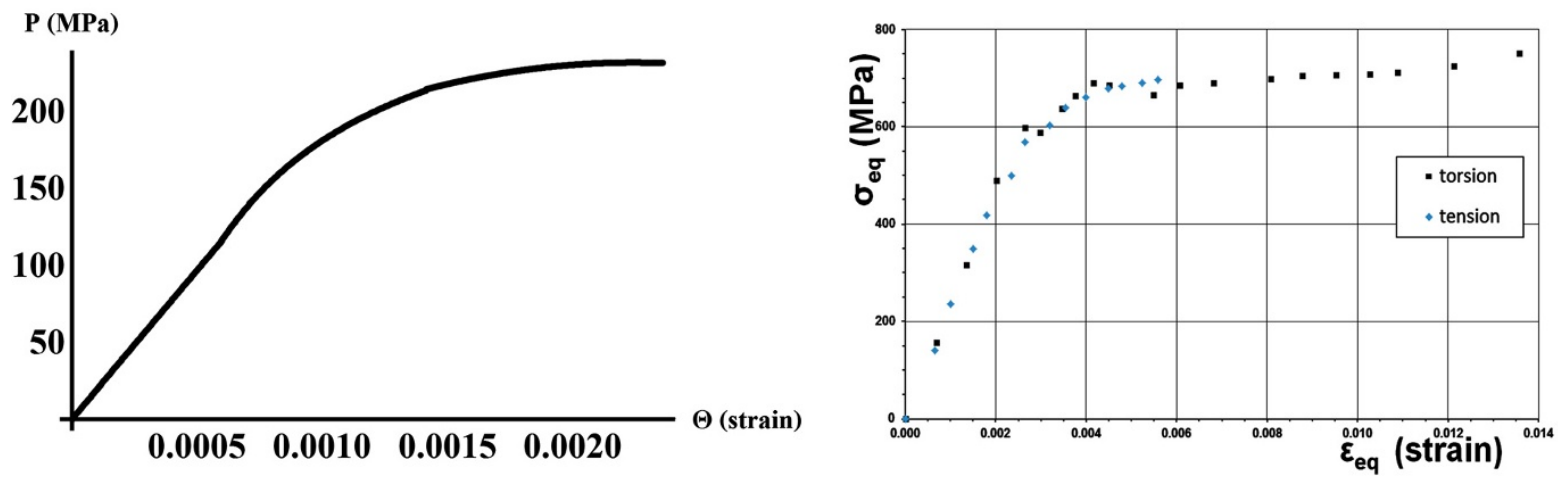

Figure $\mathbf{2}$ Theoretically predicted constitutive response and associated experimental data for En8 steel in various engineering spaces. (a) $\sigma_{1}=\sigma_{1}\left(\varepsilon_{1}\right)$, (b) $p=p(\Theta)$, (c) $\sigma_{\text {eq }}=\sigma_{\text {eq }}\left(\varepsilon_{\text {eq }}\right)$ in torsion, (d) $\sigma_{\text {eq }}=\sigma_{\text {eq }}\left(\varepsilon_{\text {eq }}\right)$ in torsion and tension. 
In addition, distinct uniaxial tension and torsion experiments were performed to obtain the constitutive behavior of the material.

\section{Constitutive equations}

Function $\sigma_{1}=\sigma_{1}\left(\varepsilon_{1}\right)$ (as defined in Ali and Hashmi (1999)) is plotted in Figure 2a along with the experimental data after transforming them to true quantities. The hydrostatic pressure $p(\Theta)$ is plotted in Figure $2 \mathrm{~b}$ as a function of the dilatation $\Theta$, as defined in Appendix 1 . The equivalent von Mises stress-strain $\sigma_{\text {eq }}\left(\varepsilon_{\text {eq }}\right)$ curve (as defined in Ali and Hashmi (1999)) is plotted in Figure 2c. For the comparison purposes, we also present all the associated experimental data from uniaxial tension and torsion in Figure 2d.

Taking into consideration the definitions of the secant bulk modulus $K_{\mathrm{S}}\left(I_{1}\right)$ being the ratio $p / \Theta$ and of the secant shear modulus $G_{\mathrm{S}}\left(J_{2}\right)$ being the ratio $\sigma_{\text {eq }} /\left(3 \varepsilon_{\text {eq }}\right)$, we can derive the graphs of these moduli as shown in Figure 3.

As it was expected, both curves show an initial flat region up to $\lambda_{1}, \lambda_{2}$. The $\lambda_{1}$ and $\lambda_{2}$ are the last points of $I_{1}$ and $\sqrt{J_{2}}$, respectively, of the linear part of the relation between the secant moduli and the strain invariants. The remaining part of the behavior can be described by exponentially decaying functions. Consequently, the total behavior can be described as

$$
\begin{aligned}
& K_{\mathrm{S}}\left(I_{1}\right)= \begin{cases}K_{\mathrm{S}}^{0} & \text { for } 0 \leq I_{1} \leq \lambda_{1} \\
K_{\mathrm{S}}^{0} e^{-m\left(I_{1}-\lambda_{1}\right)} & \text { for } I_{1}>\lambda_{1}\end{cases} \\
& G_{\mathrm{S}}\left(J_{2}\right)= \begin{cases}G_{\mathrm{S}}^{0} & \text { for } 0 \leq \sqrt{J_{2}} \leq \lambda_{2} \\
G_{\mathrm{S}}^{0} e^{-n\left(\sqrt{J_{2}}-\lambda_{2}\right)} & \text { for } \sqrt{J_{2}}>\lambda_{2}\end{cases}
\end{aligned}
$$

The reason of using $\sqrt{J_{2}}$ instead of $J_{2}$ is for ensuring that our variable quantities (i.e., $I_{1}$ and $\sqrt{J_{2}}$ ) have the same units. The derivation of the detailed form of Equation (4) via Equation (7) is described in (Andrianopoulos and Boulougouris 2004). The resulting equations are as follows: where $q, n$, and $m$ are parameters that must be defined from regression analysis of Equation (8) with experimental data.

Plots of constitutive Equations (8) are shown in Figure 4. The necessary parameters and constants are shown in Tables 1 and 2. The strong dependence of $K_{\mathrm{S}}$ on $I_{1}$ and $G_{\mathrm{S}}$ on $J_{2}$, respectively, is evident. The respective dependence of $K_{\mathrm{S}}$ on $J_{2}$ and $G_{\mathrm{S}}$ on $I_{1}$ is weak, but exists.

\section{The failure boundary}

In order to plot the failure boundary in stress space, the calculation of critical values of the elastic strain energy densities $T_{\mathrm{D}, 0}$ and $T_{\mathrm{V}, 0}$ is needed. From Figure $2 \mathrm{c}$ of the torsion experiment, $T_{\mathrm{D}, 0}$ is evaluated by elastic unloading, identical to that shown in Figure 1b. The obtained value is $T_{\mathrm{D}, 0}=1.24 \mathrm{MPa}$. As triaxial tension data do not exist, following the procedure of Appendix 1, we obtain $T_{\mathrm{V}, 0}=0.16 \mathrm{MPa}$. Here, the evolutionary continuity of the volumetric expansion part of deformation was enforced by keeping the Poisson ratio constant. The failure surface is obtained in the strain space $\left(I_{1}, \sqrt{J_{2}}\right)$ by integrating Equation (5) for some arbitrary simple stretching paths, i.e., $\sqrt{J_{2}}=s \cdot I_{1}$ where $s>0$. This way critical pairs $\left(I_{1,0}, \sqrt{J_{2,0}}\right)$ are obtained. The failure surface (Figure 5) in the Haig-Westergaard stress space $(\xi, \rho)$ can be obtained through the transformation equations (Manolopoulos 2009):

$$
\xi=\sqrt{3} K_{\mathrm{S}} I_{1}, \quad \rho=\frac{3 \sqrt{2}}{1+v} G_{\mathrm{S}} \sqrt{J_{2}}
$$

where $v$ is the Poisson ratio of the material.

\section{Results for combined loading paths}

For this specific material, there were not any experimental data from the compression tests, so the failure box is plotted only in the first quadrant (Figure 5) and the 'sheets' of $K_{\mathrm{S}}\left(I_{1}, J_{2}\right)$ and $G_{\mathrm{S}}\left(I_{1}, J_{2}\right)$ in Figure 4 are shown for positive values of $I_{1}$ only. The results of the two series of experiments are presented in Table 3.

The respective failure points are shown in Figure 5, along with the loading paths from Table 1 . In the same figure, solid, red $\left(T_{\mathrm{D}}=T_{\mathrm{D}, 0}\right)$, and green $\left(T_{\mathrm{V}}=T_{\mathrm{V}, 0}\right)$ lines represent the bounds of the failure surface.

$$
\left.\begin{array}{l}
K_{\mathrm{S}}\left(I_{1}, J_{2}\right)=K_{\mathrm{S}}^{0}\left(e^{-m\left(I_{1}-\lambda_{1}\right)}-1\right)\left[1-q G_{\mathrm{S}}^{0} \lambda_{2}^{2}+q G_{\mathrm{S}}^{0}\left(J_{2}+\frac{2-2 e^{-n\left(\sqrt{J_{2}}-\lambda_{2}\right)}\left(1+n \sqrt{J_{2}}\right)+n\left(-n J_{2}+\lambda_{2}\left(2+n \lambda_{2}\right)\right)}{n^{2}}\right)\right] \\
G_{\mathrm{S}}\left(I_{1}, J_{2}\right)=\frac{1}{4 m^{2}} G_{\mathrm{S}}^{0}\left(-1+e^{-n\left(\sqrt{J_{2}}-\lambda_{2}\right)}\right)\left[\begin{array}{l}
m^{2}\left(4-q K_{\mathrm{S}}^{0} \lambda_{1}^{2}\right)+q m^{2} K_{\mathrm{S}}^{0} I_{1}^{2}+ \\
K_{\mathrm{S}}^{0}\left(2-2 e^{-m\left(I_{1}-\lambda_{1}\right)}\left(1+m I_{1}\right)+m q\left(-m I_{1}^{2}+\lambda_{1}\left(2+m \lambda_{1}\right)\right)\right)
\end{array}\right]
\end{array}\right\}
$$



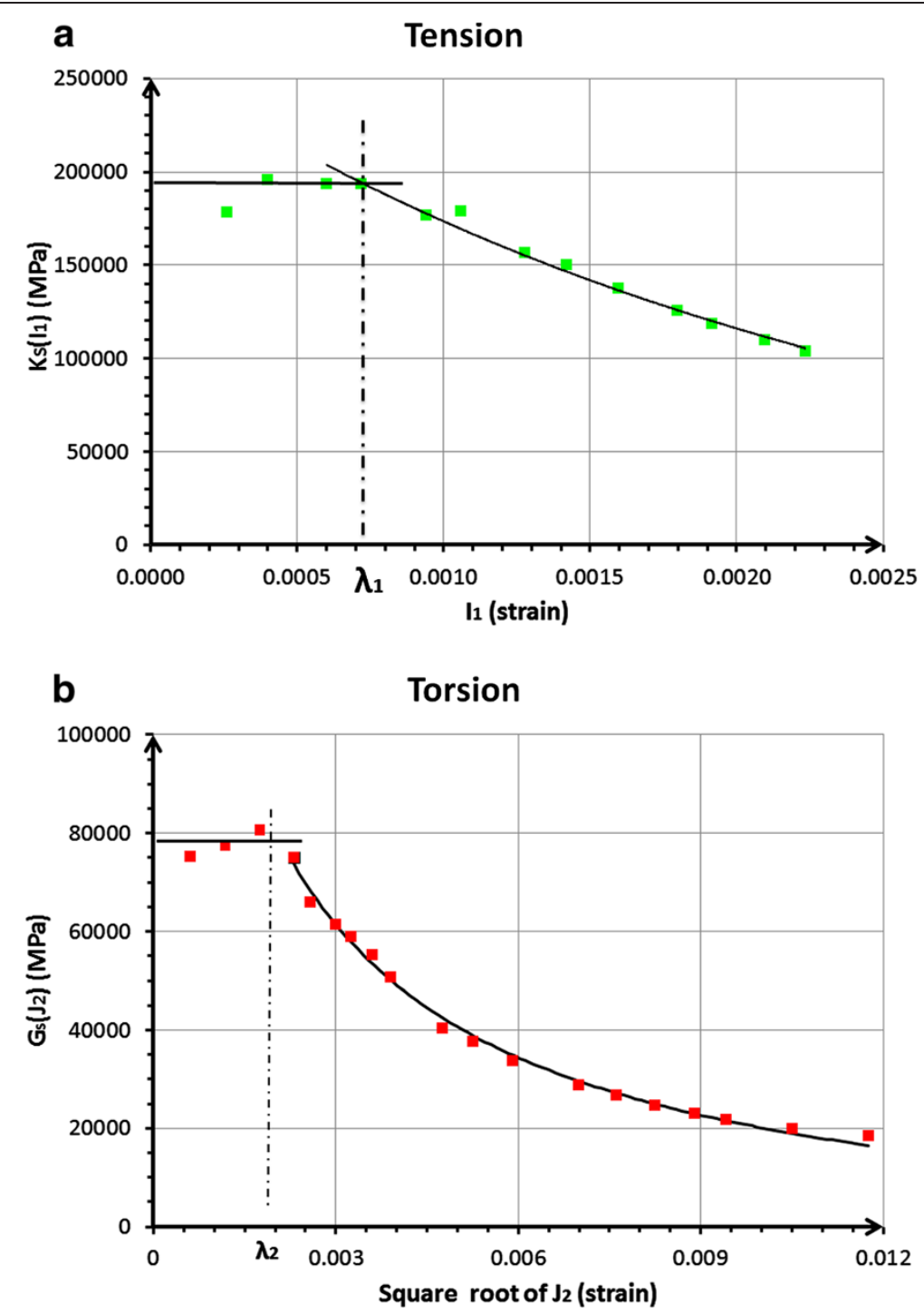

Figure 3 The two-branched functions. (a) $K_{S}\left(I_{1}\right)$ and (b) $G_{S}\left(J_{2}\right)$.

It is clear that the failure points $\mathrm{L}, \mathrm{K}, \mathrm{F}, \mathrm{J}$, and $\mathrm{E}$ belong to the curve $T_{\mathrm{D}}=T_{\mathrm{D}, 0}$ (red one), and point $\mathrm{D}$ belongs to the curve $T_{\mathrm{V}}=T_{\mathrm{V}, 0}$ (green one). The predictions of the $\mathrm{T}$-criterion are quite satisfactory.

\section{Application to Alcoa aluminum 24S-T alloy}

According to Marin (1948), thin-walled tubular specimens, made from a fully heat-treated aluminum alloy (Alcoa 24S-T), were subjected to combined tension and torsion so that the ratio of principal stresses $\sigma_{2} / \sigma_{1}$ is kept constant for each experiment and ranged from zero (uniaxial tension) to -1.0 (torsion). The test procedure consisted in applying simultaneously torsion and tension loads of predetermined amounts corresponding to a selected value of nominal principal stress ratio $\left(\sigma_{2} / \sigma_{1}\right)$. In order to find the failure surface for this material, the procedure described in 'Application to En8 steel' section is repeated and the results are presented in the sequel. In the present case, respective Equation (7) is chosen to have the following form:

$$
\begin{aligned}
& K_{\mathrm{S}}\left(I_{1}\right)= \begin{cases}K_{S}^{0} & \text { for } 0 \leq I_{1} \leq \lambda_{1} \\
a I_{1}^{b} & \text { for } I_{1}>\lambda_{1}\end{cases} \\
& G_{\mathrm{S}}\left(J_{2}\right)= \begin{cases}G_{\mathrm{S}}^{0} & \text { for } 0 \leq \sqrt{J_{2}} \leq \lambda_{2} \\
c\left(\sqrt{J_{2}}\right)^{\mathrm{h}} & \text { for } \sqrt{J_{2}}>\lambda_{2}\end{cases}
\end{aligned}
$$



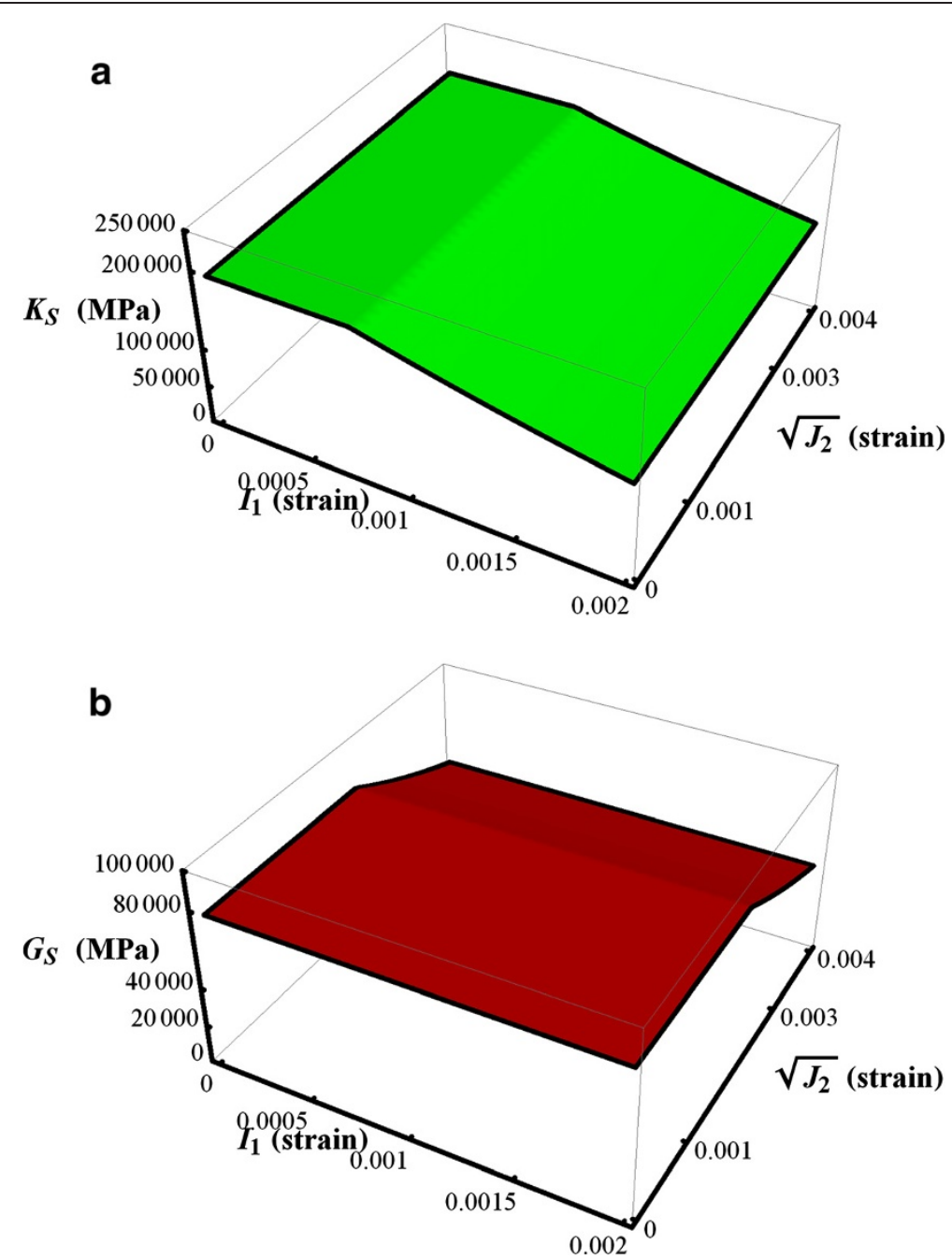

Figure 4 Constitutive equations for En8 steel. (a) $K_{S}\left(I_{1}, \sqrt{J_{2}}\right)$ and (b) $G_{S}\left(I_{1}, \sqrt{J_{2}}\right)$.

These equations are different from those of steel Equation (7) giving quite adequate agreement with experimental data through nonlinear regression. This difference in the form of fitting functions does not affect the application of T-criterion, which can be applied independently of the form of Equation (4). The derivation of the detailed form of Equation (4) via Equation (10) is described in (Andrianopoulos and Manolopoulos 2012). So,

$$
\left.\begin{array}{l}
K_{\mathrm{S}}\left(I_{1}, J_{2}\right)=a I_{1}^{b} \frac{\left[2+h+2 c q\left(\left(\sqrt{J_{2}}\right)^{2+h}-\lambda_{2}^{2+h}\right)\right]}{2+h} \\
G_{\mathrm{S}}\left(I_{1}, J_{2}\right)=c\left(\sqrt{J_{2}}\right)^{h} \frac{\left[4+2 b+a q\left(I_{1}^{2+b}-\lambda_{1}^{2+b}\right)\right]}{2(2+b)}
\end{array}\right\}
$$

The necessary parameters and constants are shown in Tables 1 and 2. The constitutive equations (Equation

\begin{tabular}{|c|c|c|c|c|c|c|c|c|c|}
\hline Material & $K_{\mathrm{S}}^{0}(\mathrm{GPa})$ & $G_{\mathrm{S}}^{0}(\mathrm{GPa})$ & $T_{\mathrm{V}, 0}(\mathrm{MPa})$ & $T_{\mathrm{D}, 0}(\mathrm{MPa})$ & $\lambda_{1}$ & $\lambda_{2}$ & $\sigma_{\mathrm{Y}}(\mathrm{MPa})$ & $\tau_{Y}(\mathrm{MPa})$ & $v$ \\
\hline Steel En8 & 193.7 & 80.4 & 0.16 & 1.24 & 0.0015 & 0.0020 & 600 & 350 & 0.3 \\
\hline Al 24S-T & 75.15 & 29.1 & 0.26 & 1.96 & 0.0019 & 0.0025 & 340 & 196 & 0.33 \\
\hline
\end{tabular}

Table 1 Properties of materials 
Table 2 Parameters and constants in Equations (8) and (11)

\begin{tabular}{llllll}
\hline Parameter & \multicolumn{5}{l}{ Constant } \\
\hline Steel En8, Equation (7) & $m$ & $n$ & $q(\mathrm{MPa})$ & & \\
& 336.7 & 277.9 & -0.00789 & & \\
Al 24S-T, Equation (10) & $a(\mathrm{MPa})$ & $b$ & $c(\mathrm{MPa})$ & $h$ & $q$ \\
& 445.33 & -0.775 & 202.54 & -0.841 & -0.042 \\
\hline
\end{tabular}

(11)) for Alcoa 24S-T are qualitatively similar to those in Figure 4 for En8 steel, but the sensitivity of $K_{\mathrm{S}}$ on $J_{2}$ and $G_{\mathrm{S}}$ on $I_{1}$ is much stronger than that in the case of steel. However, a critical difference in the behavior of the two materials is that in the case of Alcoa 24S-T, the Poisson ratio varied in order to satisfy the continuation of volume expansion $\Theta$. Here, Poisson ratio took the values $v=0.33$ in the linear elastic area, $v_{\mathrm{L}}=0.34$ in the nonlinear elastic area, and $v_{\mathrm{H}}=0.36$ in the hardening area.

The failure surface for 24S-T aluminum alloy in the Haig-Westergaard stress space $(\mathcal{\xi}, \rho)$ is shown in Figure 6 (gray area) along with the given experimental loading paths.

It is clear that the failure points $\mathrm{B}, \mathrm{C}, \mathrm{D}, \mathrm{E}$, and $\mathrm{F}$ belong to the curve $T_{\mathrm{D}}=T_{\mathrm{D}, 0}$ (red one) while the failure point A (uniaxial tension) belongs to the curve $T_{\mathrm{V}}=T_{\mathrm{V}, 0}$ (green one). The predictions of the T-criterion are quite satisfactory. All required parameters and constants for both materials are summarized in Tables 1 and 2 .

\section{Conclusions}

In the present work, a careful analysis based on experimental data from Ali and Hashmi (1999) and Marin (1948) is performed by applying the T-criterion. The underlined physical principle is that a material fails because it cannot store more elastic strain energy for the
Table 3 Combined tension-torsion data for En 8 steel

\begin{tabular}{llllll}
\hline Path & Experiment & \multicolumn{4}{l}{ Maximum values at failure } \\
\cline { 3 - 6 } & & $\boldsymbol{\sigma}_{\text {eq }}(\mathbf{M P a})$ & $\boldsymbol{\varepsilon}_{\text {eq }}$ & $\boldsymbol{p}(\mathbf{M P a})$ & $\boldsymbol{\Theta}$ \\
\hline OD & Tension & 693 & 0.0060 & 231 & 0.0024 \\
OBF & $50 \% \sigma_{Y} \rightarrow$ torsion & 725 & 0.0137 & 121 & 0.0008 \\
OCE & $75 \% \sigma_{Y} \rightarrow$ torsion & 735 & 0.0136 & 181 & 0.0021 \\
OL & Torsion & 745 & 0.0140 & 0 & 0 \\
ONJ & $50 \% \tau_{Y} \rightarrow$ tension & 702 & 0.0135 & 171 & 0.0012 \\
OMK & $75 \% \tau_{Y} \rightarrow$ tension & 710 & 0.0138 & 111 & 0.0005 \\
\hline
\end{tabular}

formation of either new plastic or elastic strains. Consequently, it fails by slip or cleavage, respectively.

This binary alternative (slip-cleavage) necessitates the introduction of two, instead of one, constitutive equations for the complete description of materials; the first dealing with normal stresses/strains and the second one dealing with shear stresses/strains. In turn, two critical quantities are required, namely, $T_{\mathrm{D}, 0}$ and $T_{\mathrm{V}, 0}$.

Even a couple of constitutive equations that predict accurately the experimental data, cannot guarantee acceptable predictions from any criterion as far as a clear distinction between 'elastic energy' and 'plastic work' is not considered. Plastic (or total) strains are results of loading and their magnitude depends on loading path (see Table 1 and for example, Bao and Wierzbicki (2004)). Proper functions of stresses and elastic strains separated into volume-shape changes give constant limits for failure.

The comparison of failure surfaces for steel (Figure 5) and aluminum (Figure 6) indicates that there are considerable qualitative differences between them. None of the two limiting lines $T_{\mathrm{V}}=T_{\mathrm{V}, 0}$ and $T_{\mathrm{D}}=T_{\mathrm{D}, 0}$ is a straight line, although this is clear only in case of aluminum. It

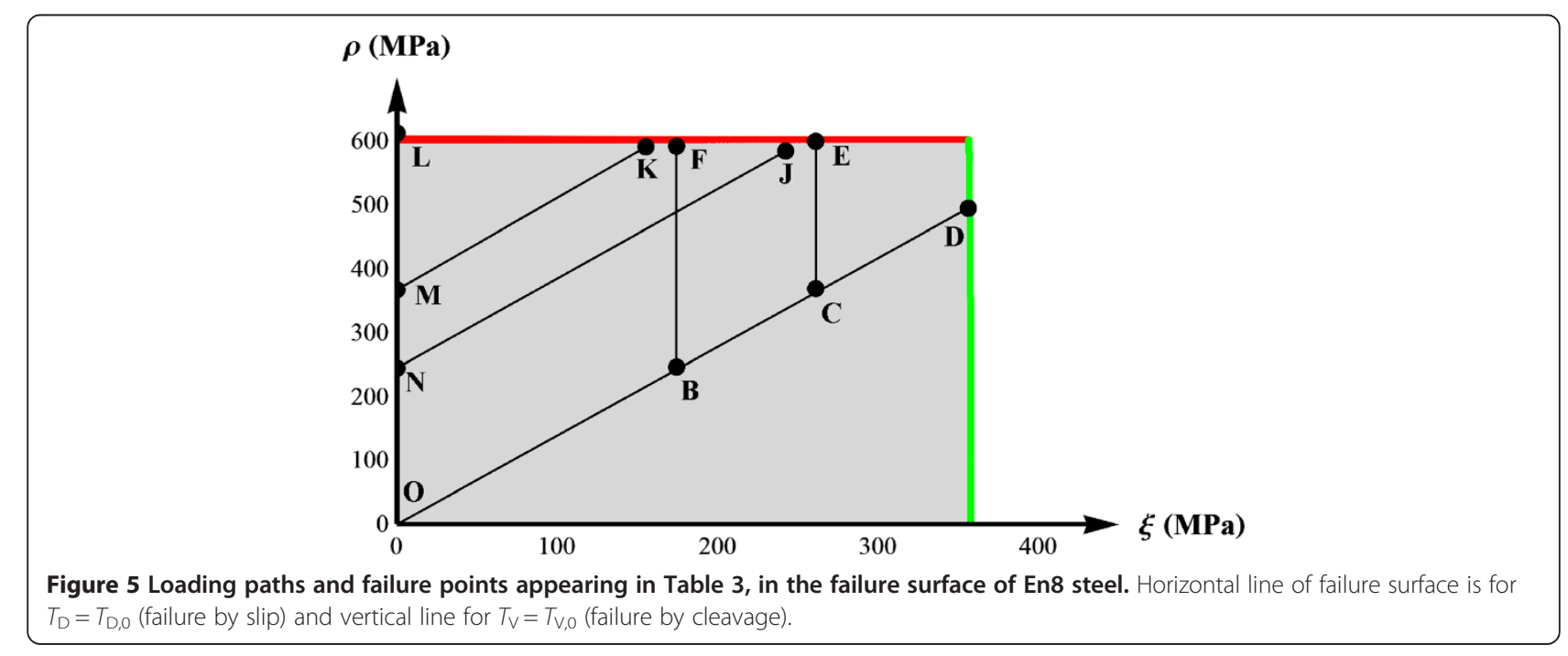




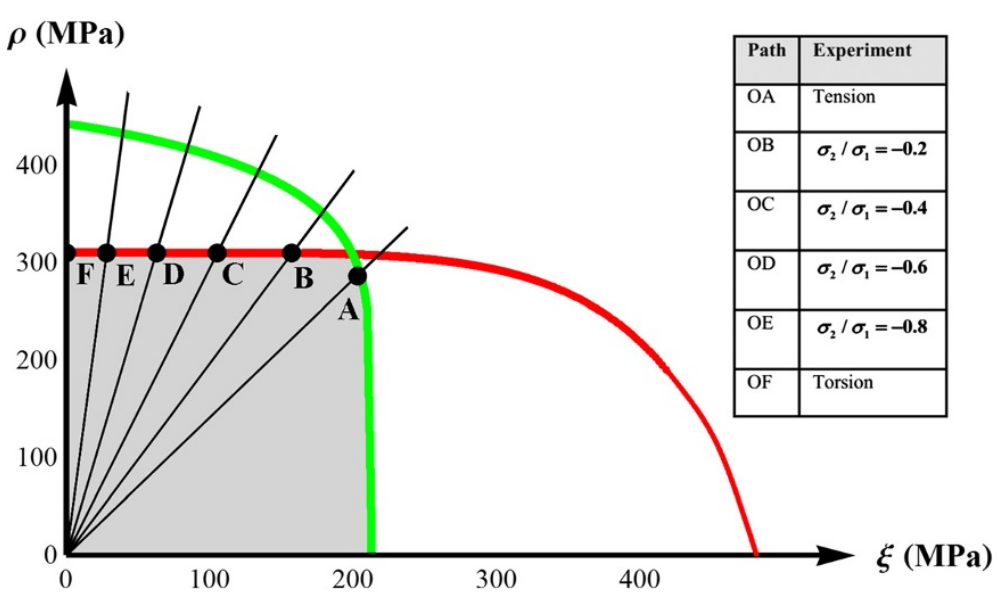

Figure 6 Loading paths and failure points in the failure box (shaded area) for aluminum 24S-T alloy.

implies that for materials like aluminum 24S-T alloy, it could be difficult to distinguish between cleavage and slip under certain combinations of stresses (area between points $A$ and $B$ in Figure 7 ). In turn, this difficulty emphasizes in an indirect way, the vital importance of having an adequate constitutive description of the material. It is obvious that single constitutive equations have no chance to describe the twin (volume-shape) character of materials. Our work aims in emphasizing that the idea of predicting the failure of a material is mainly equivalent to the problem of deriving the proper constitutive equations.

\section{Appendix 1: Derivation of curve $p=f(\Theta)$}

The standard method for obtaining the elastically stored strain energy is computing the area under the stress strain area that is bounded to the right by the unloading path. The total strain energy density, $T$, can be written in

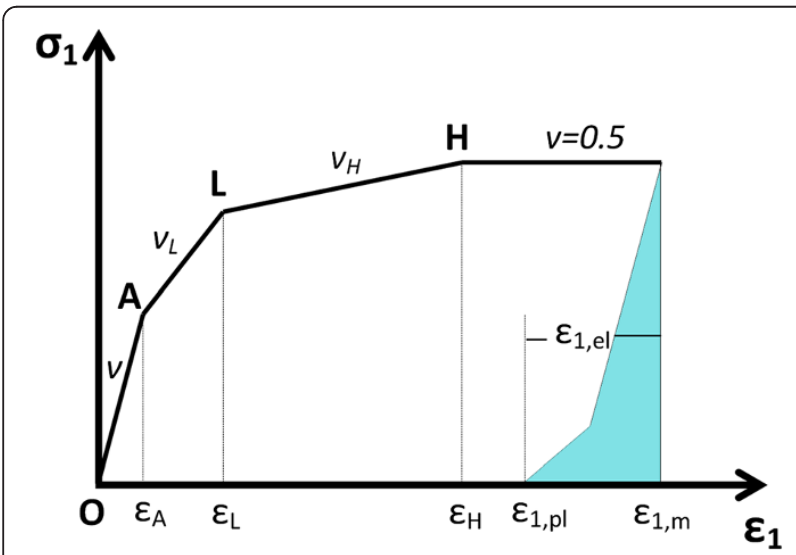

Figure 7 Qualitative curve $\sigma_{1}=\sigma_{1}\left(\varepsilon_{1}\right)$ in the most general case of a material. terms of the conjugate pairs of principal stresses and strains as follows:

$$
T=\frac{1}{2} \sum_{i=1}^{3} \int_{0}^{\sigma_{i}} \sigma_{i}\left(\varepsilon_{i}\right) d \varepsilon_{i}
$$

resulting in both volume and shape changes. The separation of total elastic strain energy density into the two respective parts $\left(T_{\mathrm{V}}, T_{\mathrm{D}}\right)$ requires distinction between elastic and plastic strains and unloading in a proper stress/strain space.

The proper space for distortional strain energy density is that of equivalent quantities, given by Equation (1) in the main body of this paper, where each shear stress corresponds to a shear strain. Then, unloading results in the evaluation of distortional elastic strain energy and plastic work.

Problems arise when the required one-to-one correspondence between stress and strain components does not hold. The most classical example of correspondence violation is uniaxial tension, where a single stress causes in an indirect way (through Poisson ratio) three strains. In addition, the proper unloading space given by Equation (2) of the main body is $(p, \Theta)$ requiring some algebra. For that we have

$$
\left.\begin{array}{l}
p=\frac{\sigma_{1}+\sigma_{2}+\sigma_{3}}{3}=\frac{\sigma_{1}}{3} \\
\Theta=\varepsilon_{1}+\varepsilon_{2}+\varepsilon_{3}=\varepsilon_{1}-v \cdot \varepsilon_{1}-v \cdot \varepsilon_{1}=(1-2 v) \varepsilon_{1}=(1-2 v)\left(\varepsilon_{1, \mathrm{el}}+\varepsilon_{1, \mathrm{pl}}\right)
\end{array}\right\}
$$

where $v$ is the Poisson ratio, $\varepsilon_{1 \text {,el }}$ the elastic part of $\varepsilon_{1}$, and $\varepsilon_{1, \mathrm{pl}}$ the plastic one.

In the most general case of a material, the curve $\sigma_{1}=\sigma_{1}\left(\varepsilon_{1}\right)$ consists of four areas marked with respect to strain as (a) linear elastic, (b) nonlinear elastic, (c) 

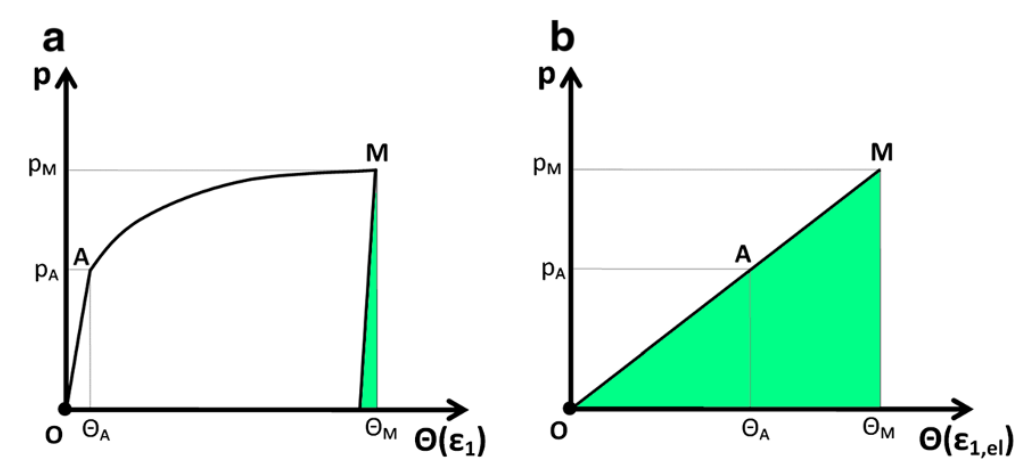

Figure 8 Hydrostatic pressure versus strain. (a) $p=f(\Theta)$ selecting total $\varepsilon_{1}$, (b) $p=f(\Theta)$ selecting elastic part of $\varepsilon_{1}$.

hardening, and (d) fully plastic, as it is typically shown in Figure 7 with line segments $(\mathrm{AL})$ and $(\mathrm{LH})$ being straight for simplicity.

It is known (Theocaris and Koroneos 1963) that Poisson ratio varies from an initial linear elastic value $v$ to a final one $v \approx 0.5$ in perfect plasticity. Consequently, the second of Equation (13) must be modified accordingly. Thus, we have

$$
\begin{cases}\Theta_{\mathrm{A}}=(1-2 v) \varepsilon, & 0<\varepsilon<\varepsilon_{\mathrm{A}} \\ \Theta_{\mathrm{L}}=\left(1-2 v_{\mathrm{L}}\right) \varepsilon, & \varepsilon_{\mathrm{A}}<\varepsilon<\varepsilon_{\mathrm{L}} \\ \Theta_{\mathrm{H}}=\left(1-2 v_{\mathrm{H}}\right) \varepsilon, & \varepsilon_{\mathrm{L}}<\varepsilon<\varepsilon_{\mathrm{H}}\end{cases}
$$

Various symbols in Equation (14) are defined in Figure 7 and $\Theta_{i}, i=A, L, H$ represents the sum of strains at each area.

Using the first of Equations (13) and (14), we can plot $p=f(\Theta)$ as shown in Figure 8a. For that, proper values for $v_{\mathrm{L}}$ and $v_{\mathrm{H}}$ satisfying strain continuation - in the present case - must be selected. If so, $p=f(\Theta)$ becomes a relationship $p\left(\sigma_{1}\right)=\Theta\left(\varepsilon_{1}, v, v_{\mathrm{L}}, v_{\mathrm{H}}\right)$ with $\sigma_{1}=\sigma_{1}\left(\varepsilon_{1}\right)$ given for the material under study and $\left(v, v_{\mathrm{L}}, v_{\mathrm{H}}\right)$ been evaluated through continuation of $\Theta$. The function $v=v\left(\varepsilon_{1}\right)$ must be a continuous function as it seems natural. It was checked that the introduction of a mean value for $v_{\mathrm{L}}, v_{\mathrm{H}}$ affects slightly the final values of $T_{\mathrm{V}, 0}$.

To plot the curve $p=f(\Theta)$, there are two alternatives at this point: either to select total $\varepsilon_{1}$ or select its elastic part $\varepsilon_{1, \mathrm{el}}$. In case of total $\varepsilon_{1}$, the results are affected by plasticity and require unloading, as indicated in Figure 8a.

On the other hand, unloading is not necessary when the elastic part of $\varepsilon_{1}$ is used. In Figure $8 \mathrm{a}, \mathrm{b}$ the green areas are equal to $T_{\mathrm{V}}=\frac{1}{2} p\left(\varepsilon_{1, \mathrm{M}}-\varepsilon_{1, \mathrm{pl}}\right)=\frac{1}{2} p \cdot \varepsilon_{1, \mathrm{el}}$.

\section{Appendix 2: Relationship between secant elastic moduli and strain invariants}

The first invariant of the strain tensor and the second invariant of the deviatoric strain tensor can be expressed as follows:

$$
I_{1}=\varepsilon_{\mathrm{kk}}, J_{2}=\frac{1}{2} e_{\mathrm{kl}} e_{\mathrm{kl}},
$$

where

$$
e_{\mathrm{kl}}=\varepsilon_{\mathrm{kl}}-\frac{1}{3} \varepsilon_{\mathrm{pp}} \delta_{\mathrm{kl}} \quad\left(\Rightarrow e_{\mathrm{kk}}=0\right)
$$

The derivatives of these invariants with respect of the strain tensor can now be expressed by

$$
\frac{\partial I_{1}}{\partial \varepsilon_{i j}}=\delta_{i j} \text { and } \frac{\partial J_{2}}{\partial \varepsilon_{i j}}=e_{k l} \frac{\partial e_{k l}}{\partial \varepsilon_{i j}} \stackrel{(13)}{=} e_{\mathrm{kl}}\left[\delta_{k l} \delta_{i l}-\frac{1}{3} \delta_{k l} \delta_{i j}\right]=e_{i j}
$$

The constitutive equation can be derived according to

$$
\begin{aligned}
\sigma_{i j} & =\frac{\partial T}{\partial \varepsilon_{i j}}=\frac{\partial T}{\partial I_{1}} \frac{\partial I_{1}}{\partial \varepsilon_{i j}}+\frac{\partial T}{\partial J_{2}} \frac{\partial J_{2}}{\partial \varepsilon_{i j}}=\frac{\partial T}{\partial I_{1}} \delta_{i j}+\frac{\partial T}{\partial J_{2}} e_{i j} \\
& =a_{1} \delta_{i j}+a_{2} e_{i j}
\end{aligned}
$$

Comparison of the last equation with the Lamé relationship between stresses and strains

$$
\sigma_{i j}=K_{S} \varepsilon_{k k} \delta_{i j}+2 G_{S} e_{i j}
$$

results to

$$
K_{\mathrm{S}} \varepsilon_{\mathrm{kk}}=K_{\mathrm{S}} I_{1}=a_{1} 2 G_{\mathrm{S}}=a_{2}
$$

Consequently, the following equation must hold:

$$
\frac{\partial a_{1}}{\partial J_{2}}=\frac{\partial\left(K_{S} I_{1}\right)}{\partial J_{2}}=\frac{\partial a_{2}}{\partial I_{1}}=2 \frac{\partial G_{S}}{\partial I_{1}}
$$

\section{Competing interests}

The authors declare that they have no competing interests.

\section{Authors' contributions}

Both authors are equally contributors to all sections of the paper. All authors read and approved the final manuscript. 
Received: 31 March 2014 Accepted: 21 August 2014

Published online: 09 October 2014

\section{References}

Ali, ARM, \& Hashmi, MSJ. (1999). Theoretical and experimental results of the elastic-plastic response of a circular rod subjected to non-proportional combined torque and tension loadings. ARCHIVE Proceedings of the Institution of Mechanical Engineers Part C Journal of Mechanical Engineering Science 1989-1996 (vols 203-210), 213(3), 251-261.

Andrianopoulos, NP. (1993). Metalforming limit diagrams according to the Tcriterion. Journal of Materials Processing and Technology, 39, 213-226.

Andrianopoulos, NP, \& Boulougouris, VC. (1994). Failure by fracture or yielding in strain hardening materials according to the T-criterion. Engineering Fracture Mechanics, 47, 639-651.

Andrianopoulos, NP, \& Boulougouris, VC. (2004). Loading path dependence of failure surfaces. Chania: 7th International Congress on Mechanics, HSTAM 2004.

Andrianopoulos, NP, \& Manolopoulos, VM. (2010). On a theoretical generalization of Coulomb criterion. Xanthi: First Greek-Ukrainian Conference on Fracture Mechanics of Materials and Structures.

Andrianopoulos, NP, \& Manolopoulos, VM. (2012). Can Coulomb criterion be generalized in case of ductile materials? An application to Bridgman experiments. International Journal of Mechanical Sciences, 54, 241-248.

Andrianopoulos, NP, Manolopoulos, VM, \& Boulougouris, VC. (2007). Considerations on failure surfaces in brittle, non linear elastic materials. Patras: 8th Int. Congress on Mechanics, HSTAM 2007.

Andrianopoulos, NP, Manolopoulos, VM, \& Dernikas, IT. (2008). Coulomb failure surfaces in ductile nonlinear elastic materials. Patras: 1st International Conference of Engineering Against Fracture.

Bao, Y, \& Wierzbicki, T. (2004). On fracture locus in the equivalent strain and stress triaxiality space. International Journal of Mechanical Sciences, 46, 81-98.

Bigoni, D, \& Piccolroaz, A. (2004). Yield criteria for quasibrittle and frictional materials. International journal of Sol and Structure, 41, 2855-2878.

Bridgman, PW. (1952). Studies in large plastic flow and fracture (1st ed.). New York: McGraw-Hill.

Christensen, RM. (1997). Yield functions/failure criteria for isotropic materials. Proceedings of the Royal Society of London, 453, 1473-1491.

Christensen, RM. (2004). A two-property yield, failure (fracture) criterion for homogeneous isotropic materials. Journal of Engineering Materials and Technology, 126, 45-52.

Christensen, RM. (2014). Failure mechanics - part I: the coordination between elasticity theory and failure theory for all isotropic materials. Journal of Applied Mechanics, 81(8), 1-7.

Drucker, DC, \& Prager, W. (1952). Soil mechanics and plastic analysis for limit design. Quarterly of Applied Mathematics, 10, 157-165.

Hencky, H. (1924). Zur Theorie plastischer Deformationen und der hierdurch im Material hervorgerufenen Nachspannungen. Zeitschrift Angewandte Mathematics and Mechanics, 4, 323-334.

Heyman, J. (1997). Coulomb's memoir on statics, an essay in the history of civil engineering. London: Imperial College Press.

Huber, MT. (1904). Die spezifische formänderungsarbeit als mass der anstrengung eines materials. Czasopismo Techniczne, 22, 81-92.

Li, QM. (2001). Strain energy density failure criterion. International Journal of Solids and Structures, 38, 6997-7013.

Mahendra, S, \& Bhawani, S. (2012). Modified Mohr-Coulomb criterion for nonlinear triaxial and polyaxial strength of jointed rocks. International Journal of Rock Mechanics and Mining Science, 51, 43-52.

Manolopoulos, VM. (2009). Contribution to the study of failure criteria on non linear elastic materials, PhD Dissertation. In Greek: National Technical University of Athens.

Marin, J. (1948). Stress-Strain relations in the plastic range for biaxial stresses (pp. 231-249). London: International Congress on Applied Mechanics.

Maxwell, C. (1937). The origins of Clerk Maxwell's electric ideas, as described in familiar letters to W. Thomson. Mathematical Proceedings of the Cambridge Philosophical Society, 32, 695-748.

Mohr, O. (1914). Abhandlungen aus demGebiete der Technischen Mechanik (2nd ed.). Berlin: Ernst.

Paul, B. (1968). In H Liebowitz (Ed.), Macroscopic criteria for plastic flow and brittle fracture in fracture (pp. 313-496). New York: Academic.

Schajer, GS. (1998). Mohr-Coulomb failure criterion expressed in terms of stress invariants. Journal of Applied Mechanics, 65, 1066-1068.
Stassi, F. (1967). Flow and fracture of materials according to a new limiting condition of yielding. Meccanica, 3, 178-195.

Theocaris, PS, \& Andrianopoulos, NP. (1982a). The Mises elastic-plastic boundary as the core region in fracture criteria. Engineering Fracture Mechanics, $16,425-432$.

Theocaris, PS, \& Andrianopoulos, NP. (1982b). The T-criterion applied to ductile fracture. International Journal of Fracture, 20, R125-R130.

Theocaris, PS, \& Koroneos, E. (1963). Stress-strain and contraction ratio curves for polycrystalline steel. Philosophical Magazine, 8(95), 1871-1893.

von Mises, R. (1913). Die mechanik der festen körper in plastisch deformierten zustand. Nachrichten Göttingen Akademie Wissenschaftliche, Math-physik, 1, 582-592.

\section{doi:10.1186/s40712-014-0016-5}

Cite this article as: Andrianopoulos and Manolopoulos: Elastic strain energy density decomposition in failure of ductile materials under combined torsion-tension. International Journal of Mechanical and Materials Engineering 2014 9:16.

\section{Submit your manuscript to a SpringerOpen ${ }^{\circ}$ journal and benefit from:}

- Convenient online submission

- Rigorous peer review

- Immediate publication on acceptance

- Open access: articles freely available online

- High visibility within the field

- Retaining the copyright to your article

Submit your next manuscript at $>$ springeropen.com 